\title{
Safety of sublingual immunotherapy started during the pollen season
}

Renato Ariano ${ }^{a}$, Cristoforo Incorvaia ${ }^{b}$ Stefania La Gruttac, Francesco Marcucci ${ }^{d}$, Giovanbattista Pajno ${ }^{e}$, Laura Sensi ${ }^{d}$, Giuseppe Di Cara ${ }^{d}$, Jochen Sieber ${ }^{f}$, Mona-Rita Yacoub ${ }^{9}$ and Franco Frati $^{\text {h }}$

aAllergy Department, ASL 1 Imperiese, Bordighera, Italy

${ }^{b}$ Allergy/Pulmonary Rehabilitation, ICP Hospital, Milan, Italy

'Environment and Health, IBM CNR, ARPA Sicilia, Palermo, Italy

${ }^{d}$ Pediatrics, University Department of Medical and Surgical Specialties

and Public Health, Perugia, Italy

${ }^{e}$ Pediatric Allergy, Universitary Hospital G. Martino, Messina, Italy

${ }^{f}$ Medical Department, Stallergenes, Kamp-Lintfort, Germany

${ }^{g}$ Allergy and Rheumatology Unit, IRCCS San Raffaele Hospital, Milan, Italy

${ }^{h}$ Medical and Scientific Department, Stallergenes, Milan, Italy

Address for correspondence: Franco Frati, Scientific and Medical Department, Stallergenes Italia,

Via Traiano 7, Milan, Italy. Tel.: +39-3355285619; Fax: +39-0270058779; frati.f@stallergenes.it

Key words: Children - Pollen allergy - Safety - Sublingual immunotherapy - Ultrarush

\section{ABSTRACT}

Background: Sublingual immunotherapy (SLIT) is safer than subcutaneous immunotherapy (SCIT) and this has lead to the reconsideration of the use of ultra-rush schedules for SLIT. The aim of this study was to assess the safety of ultrarush SLIT in pollen-allergic children according to different timing of administration in relation to the pollen season.

Methods: In total, 34 children with pollen-induced rhinitis and 36 with pollen-induced asthma and rhinitis, were enrolled and assigned to three study groups: group 1 ( $n=17$ patients): conventional pre-seasonal-SLIT treatment; group 2 ( $n=23$ patients), seasonal SLIT ended before the pollen seasonal peak; group 3 ( $n=30$ patients), SLIT began after the pollen seasonal peak and ended after the pollen season. SLIT was performed using extracts from Stallergenes (Antony, France) and following an ultra-rush schedule, consisting in four doses at a 30-min intervals, and maintenance treatment by administering the top dose three times a week.

Results: In all, 54 adverse events (AEs) were reported: 12 in nine patients in group $1(9 / 17,52.9 \%), 22$ in 14 patients in group $2(14 / 23,60.9 \%)$, and 20 in 13 patients in group $3(13 / 30,43.3 \%)$. No statistically significant differences were found between the three groups. Local AEs (oral itching and burning) were short lasting and selfresolving. Systemic AEs were also mild, except for a case of asthma, which lasted 5 days, in a patient from group 1 . There were no severe reactions, and none of the patients dropped out.

Conclusions: This study suggests that SLIT with pollen extracts may be safely started at the beginning and also during the pollen season, with a tolerability profile comparable to the conventional pre-seasonal SLIT.

\section{Introduction}

Sublingual immunotherapy (SLIT) is currently considered a valid alternative to traditional subcutaneous immunotherapy (SCIT) in the treatment of allergic rhinitis and asthma ${ }^{1}$. The main advantage of SLIT compared to SCIT concerns its safety, and was a major factor for its acceptance ${ }^{2}$. The safety profile of SLIT 
has lead its use to be considered in very young children. Whereas the 1998 WHO Position paper did not recommend immunotherapy in children younger than 6 years of age $^{3}$, subsequent studies have shown that SLIT may be safely used in children aged 3-6 years ${ }^{4,5}$.

Other safety concerns focus on performance of rush schedules and administration of immunotherapy during the pollen season. Regarding the former, rush-SCIT has a high risk of reactions and is therefore recommended only for research purpose ${ }^{3}$, while ultra-rush SLIT - reaching the maintenance dose in a very short time - showed a safety profile comparable to conventional schedules ${ }^{6-9}$. In particular, in children schedules as short as $20 \mathrm{~min}$ have been well-tolerated ${ }^{7}$. Regarding the time for specific immunotherapy administration in relation to pollen seasonal exposure, a reduced maintenance SCIT dose is recommended ${ }^{3}$, while data for SLIT are missing ${ }^{10}$.

This study was aimed at evaluating the safety of ultra-rush SLIT in children, which was started at different times in the pollen season, to compare the number and the severity of adverse events (AEs), and to investigate whether higher allergen exposure increases the risk of AEs.

\section{Patients and methods}

In all, 70 patients aged $4-17$ years and sensitised to grass or tree pollens were enrolled in the study; 34 subjects had only rhinitis, 36 had asthma and rhinitis. Exclusion criteria were: sensitisation to other allergens causing symptoms in the same period of the studied allergens (i.e. cypress, Parietaria, and olive tree pollen, and perennial allergens), previous SCIT or SLIT during the last 3 years and common contraindications to immunotherapy $^{3}$. SLIT was performed by administering extracts (standardised by Index of Reactivity (IR)) containing tree pollens (birch, alder and hazel trees) or grass pollens (cocksfoot, meadow grass, rye-grass, sweet vernal grass and timothy) supplied by Stallergenes (Antony, France), by an ultra-rush schedule consisting of four doses (30, 90, 150, 300 IR) at 30-min intervals, with a total time of $90 \mathrm{~min}$ to reach the maximum dose. Afterwards, maintenance SLIT was carried out by administering the 300-IR dose three times a week for 16 weeks. The equivalent amount of major allergen in the maintenance dose was $24 \mu \mathrm{g}$ of Phl p 5 for grass pollen and $49.2 \mu \mathrm{g}$ of Bet $\mathrm{v} l$ for birch pollen.

According to the period of SLIT administration in relation to air pollen concentration, subjects were divided into three groups: group 1, SLIT started and ended before the pollen season, group 2, SLIT started at the beginning of the pollen season and ended before the pollen peak, and group 3, SLIT started after the pollen peak and ended after the end of pollen season. The pollen peak period was defined as pollen grain counts higher than $50 / \mathrm{m}^{3}$ in the air ${ }^{11}$. Patients were sequentially assigned into the three study groups according to the time of their presentation at the centres. The study was approved by the ethical committees of the participating centres and was conducted in accordance with the latest version of the Declaration of Helsinki, and the principles of Good Clinical Practice. All patients, or their relatives in the case of minors, gave a written informed consent.

Safety was assessed by means of the number and the severity of AEs for each patient, and the proportion of patients with AEs in each group. During the ultra-rush build-up phase, AEs were registered directly by the physician in charge, while during the maintenance phase, patients or their parents (depending on patient's age) reported all AEs in clinical diaries. Diaries were reviewed by physicians at each of the four visits planned in the study, and all written material was checked by a Clinical Research Organization.

Concerning previous drug treatment, all symptomatic drugs used prior to the start of the study were withdrawn - only the following anti-allergic drugs were allowed: oral loratadine or cetirizine, oral prednisolone, nasal and ocular azelastine, nasal fluticasone, inhaled $\beta_{2}$-agonists and inhaled corticosteroids.

\section{Statistical analysis}

The three groups were compared by means of $2 \times 2$ contingency tables. Considering the low number of samples for some boxes of the tables $(\leq 10)$, Fisher's exact test was used because it considers all the possible cell combinations that would still result in the marginal frequencies. This test is appropriate when dealing with small counts, and is exact because it uses the exact geometric distribution rather than the approximate chi-square distribution to compute the $p$-value. Significance of the test was set at a $p$-value lower than 0.05 .

\section{Results}

The main characteristics of the three groups are reported in Table 1. Three patients (one in group 1 and two in group 2) were treated with tree pollen extract and 67 (16 in group 1, 21 in group 2, and 30 in group 3) with grass pollen extract. A total of 54 AEs were reported: 12 AEs were reported in nine patients in group 1, 22 AEs were reported in 14 patients in group 2, and 20 AEs were reported in 13 patients 
Table 1. Characteristics of patients in the three groups

\begin{tabular}{|c|c|c|c|}
\hline & Group 1 & Group 2 & Group 3 \\
\hline Number of patients & 17 & 23 & 30 \\
\hline Kind of sensitisation & $\begin{array}{l}15 \text { grass pollen, } 1 \text { birch } \\
\text { pollen, } 1 \text { grass pollen } \\
\text { and horse epithelium }\end{array}$ & $\begin{array}{l}19 \text { grass pollen, } 2 \text { birch } \\
\text { pollen, } 2 \text { grass pollen } \\
\text { and plane tree pollen }\end{array}$ & $\begin{array}{l}28 \text { grass pollen, } 1 \text { grass } \\
\text { pollen and poplar } \\
\text { pollen, } 1 \text { grass pollen } \\
\text { and Cladosporium }\end{array}$ \\
\hline Patients with rhinitis & 12 & 16 & 22 \\
\hline $\begin{array}{l}\text { Patients with rhinitis and } \\
\text { asthma }\end{array}$ & 5 & 7 & 8 \\
\hline Severity of rhinitis & $\begin{array}{r}11 \text { severe persistent, } 6 \\
\text { moderate persistent }\end{array}$ & $\begin{array}{r}15 \text { severe persistent, } 8 \\
\text { moderate persistent }\end{array}$ & $\begin{array}{l}19 \text { severe persistent, } 11 \\
\text { moderate persistent }\end{array}$ \\
\hline Severity of asthma & $\begin{array}{l}2 \text { slight intermittent, } 3 \\
\text { moderate persistent }\end{array}$ & $\begin{array}{r}3 \text { slight intermittent, } 4 \\
\text { moderate persistent }\end{array}$ & $\begin{array}{r}4 \text { slight intermittent, } 4 \\
\text { moderate persistent }\end{array}$ \\
\hline
\end{tabular}

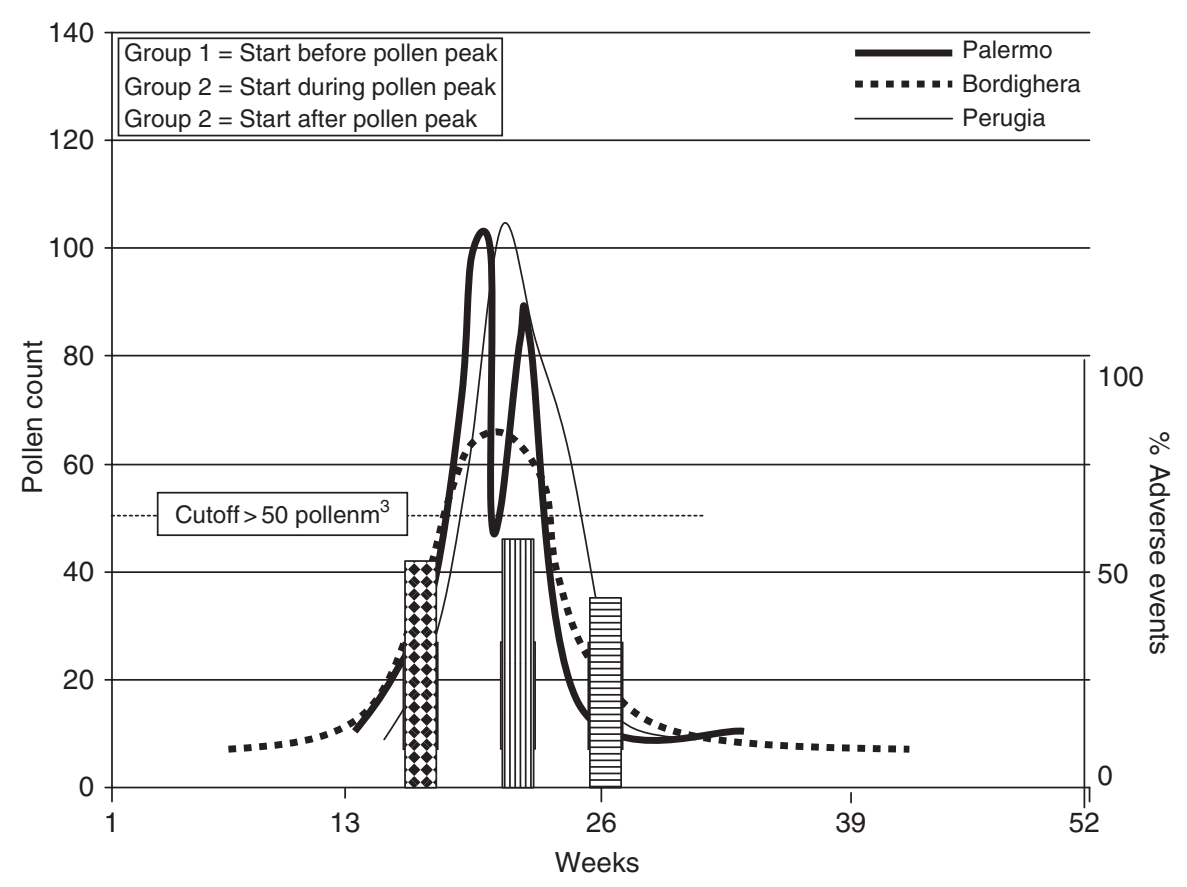

Figure 1. Rate of adverse events to SLIT according to pollen counts

in group 3. Figure 1 shows the rate of AEs according to the air pollen counts. None of differences between the three groups was statistically significant using Fisher's exact test. Local AEs, consisting of oral itching and burning of the tongue, were short lasting and did not require any treatment or SLIT dosage adjustments. Systemic AEs were also mild and self-resolving, including six cases of asthma, which resolved within a day following administration of inhaled bronchodilators, except for one case in one patient in group 1, which lasted 5 days and required inhaled corticosteroids. There were no severe reactions, and none of the patients dropped out. AEs details are reported in Table 2.

\section{Discussion}

Safety of SLIT is a major issue, and many European countries currently prefer to use SLIT rather than SCIT in order to avoid systemic reactions. During the last decade, several studies have confirmed a high safety profile for SLIT. The first meta-analysis on SLIT showed no anaphylactic reactions ${ }^{10}$, and even though such reactions have been reported recently, they should be treated with caution: in one case a mixture of nonstandardised allergens (not recommended in consensus documents) had been used, while a second case was not indicative of anaphylaxis ${ }^{12}$. The only consistent case concerning an anaphylactic reaction was one due to 
Table 2. Number and characteristics of the AEs evaluated

\begin{tabular}{llll}
\hline & Group 1 & Group 2 & Group 3 \\
\hline Sample size & 17 & 23 & 30 \\
Patients with AE & $9(53 \%)$ & $14(61 \%)$ & $13(43 \%)$ \\
Number of AE & 12 & 22 & 20 \\
Systemic side-effects & & & \\
$\quad$ Abdominal pain & 0 & 1 & 0 \\
Urticaria & 0 & 2 & 1 \\
Eczema & 0 & 0 & 1 \\
Conjunctivitis & 1 & 1 & 0 \\
Rhinitis & 0 & 2 & 2 \\
Nasal itching & 0 & 1 & 0 \\
Wheezing & 1 & 2 & 3 \\
Headache & 2 & 1 & 2 \\
Fever & 1 & 2 & 1 \\
$\quad$ Faintness & 0 & 1 & 1 \\
Local side-effects & & & \\
$\quad$ Burning/swelling & 5 & 3 & 4 \\
$\quad$ of the tongue & & & \\
Oral itching & 2 & 6 & 5 \\
\hline
\end{tabular}

a latex extract, but it is of note that in a double-blind placebo-controlled study conducted in 26 children with the same extract, no AE related to SLIT treatment was described ${ }^{13}$.

Nevertheless, the possibility of systemic reactions to SLIT should not be ruled out. When considering AEs, the different way of administering SCIT and SLIT (by physicians in a clinic and by patients at home, respectively) should be taken into account. Concerning SLIT, it may be preferable to reach the maintenance dose under medical surveillance rather than by the traditional progressive build-up period. During the traditional build-up, which lasts 10-15 days, the patient may not notice possible early signs of systemic reactivity that may lead to the more serious AEs.

In the present study, an ultra-rush schedule of 90 minutes to reach a maintenance high dose was used. No severe systemic reaction occurred, and there was an overall local-reaction rate of $37 \%$, which is comparable to rates observed using conventional schedules ${ }^{4,10}$. The study took place in real-life- a double-blind placebo-controlled trial was not performed. Because allergen extracts were already available, patients entered the study as they presented at participating centres and immediately began SLIT. The aim of the study was to assess the safety of SLIT under different conditions using an ultra-rush schedule; this made it less important to have a placebo control. Meta-analyses and systematic reviews are available that compare the safety of SLIT and placebo treatment ${ }^{10}$.
The safety assessment was aimed at defining the feasibility of starting immunotherapy during the pollen season. Reaching the maintenance dose in a few hours using ultra-rush protocols was the purpose of introducing a new preparations in tablet form, by which means SLIT can be started immediately with the maintenance dose. Efficacy studies are currently planned, using a new preparation in tablet form. The main objective of our study was to verify whether administering SLIT at different times in relation to seasonal pollen exposure might have some effect on a patient's tolerability. In particular, one group of patients commenced SLIT at the beginning of the pollen season and another group commenced SLIT when the peak of exposure to seasonal pollen had already passed. When these two groups were compared with the group receiving pre-seasonalSLIT (i.e., the usual practice), no significant differences in safety were found. Natural exposure to pollen is believed (for example because of the priming effect) to increase allergic reactivity, but the lack of significant difference in AEs in the three groups we studied suggests that this does not influence the reactivity to allergen extract administered by SLIT.

The limitations of this study are its relatively small size and the lack of evaluation of clinical efficacy.

These findings may support a recent proposal to initiate grass pollen SLIT directly with the maintenance dose ${ }^{14,15}$, provided that the first administration is carried out under medical control. The feasibility of beginning SLIT just before, or even during the pollen season, should obviously be explored in controlled trials which assess clinical efficacy. In fact, most studies on SLIT in pollinosis used pre-seasonal protocols starting 4 months prior to the pollen season ${ }^{10,16}$. Only one study has used an ultra-rush protocol administering SLIT at the beginning of the pollen season in patients allergic to cypress pollen, and this achieved a significantly better clinical outcome compared with placebo ${ }^{9}$. This finding has lead Bousquet to hypothesise that the rapid effect of SLIT is related to early immunologic actions such as downregulation of mast cells, production of interleukin (IL)-10 which induce T-cell anergy and cell desensitisation ${ }^{17}$.

\section{Conclusions}

SLIT with pollen extracts may be started at the beginning of the pollen season and even during the pollen peak with a tolerability profile comparable to the conventional pre-seasonal SLIT and no risk of severe systemic reactions. This highlights a further difference between SLIT and SCIT, but due to the relatively small size of this study larger controlled trials are 
needed to exhaustively evaluate the adequacy of such an approach.

\section{Acknowledgements}

Declaration of interest: This study was sponsored by Stallergenes, Italia, which had a role in planning the study and in preparing and reviewing the article. R.A., S.L.G., F.M., G.P., L.S., G.D.C. and M.-R. Y. have no relevant conflicts of interest with regards to the contents of this article.

C. I. is a scientific consultant for Stallergenes, Milan, Italy. J.S. is Medical Director, Stallergenes, KampLintfort, Germany. F. F. is Medical and Scientific Director, Stallergenes, Milan, Italy.

\section{References}

1. Passalacqua G, Durham SR. Allergic rhinitis and its impact on asthma update: allergen immunotherapy. J Allergy Clin Immunol 2007;119:881-91

2. Canonica GW, Passalacqua G. Noninjection routes for immunotherapy. J Allergy Clin Immunol 2003;111:437-48

3. WHO Position Paper. Allergen immunotherapy: therapeutic vaccines for allergic diseases. Allergy 1998;53(Suppl 54):4-30

4. Fiocchi A, Pajno GB, La Grutta S, et al. Safety of sublingualswallow immunotherapy in children aged less than 6 years. Ann Allergy Asthma Immunol 2005;95:254-8

-5. Di Rienzo V, Minelli M, Musarra A, et al. Post-marketing survey on the safety of sublingual immunotherapy in children below the age of 5 years. Clin Exp Allergy 2005;35:560-4

6. Rossi RE, Monasterolo G. A pilot study of feasibility of ultra-rush (20-25 min) sublingual-swallow immunotherapy in 679 patients
(699 sessions) with allergic rhinitis and/or asthma. Int J Immunopathol Pharmacol 2005;18:277-85

7. Gammeri E, Arena A, D'Anneo R, et al. Safety and tolerability of ultra-rush (20 min) sublingual immunotherapy in patients with allergic rhinitis and/or asthma. Allergol Immunopathol 2005;33:221-3

8. Tripodi S, Di Rienzo Businco A, Benincori N, et al. Safety and tolerability of ultra-rush induction, less than one hour, of sublingual immunotherapy in children. Int Arch Allergy Immunol 2006;139:149-52

9. Vervloet D, Birnbaum J, Laurent P, et al. Safety and efficacy of Juniperus ashei sublingual-swallow ultra-rush pollen immunotherapy in cypress rhinoconjunctivitis. Int Arch Allergy Immunol 2007;142:239-46

10. Wilson DR, Torres-Lima M, Durham S. Sublingual immunotherapy for allergic rhinitis: systematic review and meta-analysis. Allergy 2005;60:4-12

11. Smith M, Emberlin J. Constructing a 7-day ahead forecast model for grass pollen at north London, United Kingdom. Clin Exp Allergy 2005;35:1400-6

12. Andrè C, Fadel R. Anaphylaxis caused by sublingual immunotherapy? Allergy 2007;62:1220-1

13. Bernardini R., Campodonico P, Burastero S, et al. Sublingual immunotherapy with a latex extract in paediatric patients: a double-blind, placebo-controlled study. Curr Med Res Opin 2006;22:1515-22

14. Dahl R, Kapp A, Colombo G, et al. Efficacy and safety of sublingual immunotherapy with grass pollen tablets for seasonal allergic rhinoconjunctivitis. J Allergy Clin Immunol 2006; 118:434-40

15. Didier A, Malling HJ, Worm M, et al. Optimal dose, efficacy, and safety of once-daily sublingual immunotherapy with a 5 -grass pollen tablet for seasonal allergic rhinitis. J Allergy Clin Immunol 2007;120:1338-45

16. Penagos M, Passalacqua G, Compalati E, et al. Meta-analysis of the efficacy of sublingual immunotherapy in the treatment of allergic asthma in pediatric patients, 3 to 18 years of age. Chest 2008;133:599-609

17. Bousquet J. Sublingual immunotherapy: from proven prevention to putative rapid relief of allergic symptoms. Allergy 2005;60:1-3

\author{
CrossRef links are available in the online published version of this paper: \\ http://www.cmrojournal.com \\ Paper CMRO-4729_3, Accepted for publication: 31 October 2008 \\ Published Online: 26 November 2008 \\ doi:10.1185/03007990802591673
}

